\title{
Focal Areas of High Signal Intensity in Children with Neurofibromatosis Type 1: Expected Evolution on MRI
}

(D) Calvez, (D) R. Levy, (D). Calvez, (D).-J. Roux, (D). Grévent, (D)Y. Purcell, (D). Beccaria, (D). Blauwblomme, (D). Grill, (D). Dufour, (D) B. Bourdeaut, (DF. Doz, (D) M.P. Robert, (D) N. Boddaert, and (D). Dangouloff-Ros

\begin{abstract}
BACKGROUND AND PURPOSE: Focal areas of high signal intensity are T2WI/T2-FLAIR hyperintensities frequently found on MR imaging of children diagnosed with neurofibromatosis type 1, often thought to regress spontaneously during adolescence or puberty. Due to the risk of tumor in this population, some focal areas of high signal intensity may pose diagnostic problems. The objective of this study was to assess the characteristics and temporal evolution of focal areas of high signal intensity in children with neurofibromatosis type 1 using long-term follow-up with MR imaging.
\end{abstract}

MATERIALS AND METHODS: We retrospectively examined the MRIs of children diagnosed with neurofibromatosis type 1 using the National Institutes of Health Consensus Criteria (1987), with imaging follow-up of at least 4 years. We recorded the number, size, and surface area of focal areas of high signal intensity according to their anatomic distribution on T2WI/T2-FLAIR sequences. A generalized mixed model was used to analyze the evolution of focal areas of high signal intensity according to age, and separate analyses were performed for girls and boys.

RESULTS: Thirty-nine patients (ie, 285 MR images) with a median follow-up of 7 years were analyzed. Focal areas of high signal intensity were found in $100 \%$ of patients, preferentially in the infratentorial white matter (35\% cerebellum, $30 \%$ brain stem) and in the capsular lenticular region (22\%). They measured $15 \mathrm{~mm}$ in $95 \%$ of cases. They appeared from the age of 1 year; increased in number, size, and surface area to a peak at the age of 7; and then spontaneously regressed by 17 years of age, similarly in girls and boys.

CONCLUSIONS: Focal areas of high signal intensity are mostly small $(<15 \mathrm{~mm})$ abnormalities in the posterior fossa or capsular lenticular region. Our results suggest that the evolution of focal areas of high signal intensity is not related to puberty with a peak at the age of 7 years. Knowledge of the predictive evolution of focal areas of high signal intensity is essential in the follow-up of children with neurofibromatosis type 1.

ABBREVIATIONS: $\mathrm{FASI}=$ focal areas of high signal intensity; $\mathrm{HH}=$ hippocampal hyperintensity; $\mathrm{NF} 1=$ neurofibromatosis type 1

$\mathbf{N}$ eurofibromatosis type 1 (NF1) is a relatively common neurocutaneous disorder, with an estimated prevalence of 1 in 3000-4000 individuals. ${ }^{1}$ The most common brain lesions in patients with NF1 $\left(60 \%-70 \%\right.$ of children $\left.{ }^{2,3}\right)$ identified on MR imaging are T2WI hyperintensities, also termed focal areas of

Received January 21, 2020; accepted after revision June 7.

From the Pediatric Radiology Department (S.C., R.L., R.C., C.-J.R., D.G., N.B., V.D.-R.) Pediatric Neurosurgery Department (K.B., T.B.), and Ophthalmology Department (M.P.R.), Hôpital Universitaire Necker-Enfants Malades, Assistance PubliqueHôpitaux de Paris, Paris, France; Paris University (R.L., C.-J.R., D.G., K.B., T.B., F.B., F.D., M.P.R., N.B., V.D.-R.), PRES Sorbonne Paris Cité, Paris, France; Institut National de la Santé et de la Recherche Médicale UA10 (R.L., C.-J.R., D.G., N.B., V.D.-R.), Paris, France; Institut Imagine (R.L., C.-J.R., D.G., N.B., V.D.-.R.), Unite Mixte de Recherche 1163, Paris, France; Radiology Department (Y.P.), Fondation Rothschild, Paris, France; Department of Pediatric and Adolescent Oncology (J.G., C.D.), Gustave Roussy Institute, Villejuif, France; Oncology Center SIREDO (Care Innovation and Research for Children, Adolescents and Young Adults with Cancer) (F.B., F.D.), Institute Curie, Paris, France. high signal intensity (FASI) or unidentified bright objects. FASI are usually considered a benign process caused by increased fluid accumulation in intramyelinic vacuoles. ${ }^{4,5}$ Their clinical significance remains largely unknown; an association between clinical symptoms in NF1 and FASI has rarely been reported, notably cognitive dysfunction when FASI involved the thalamus and basal ganglia. ${ }^{6-10}$

To date, the literature suggests that these lesions can vary in size and number with time, though published articles have variable

Please address correspondence to Volodia Dangouloff-Ros, MD, MSc, AssistancePublique Hôpitaux de Paris, Hôpital Universitaire Necker-Enfants Malades, Department of Pediatric Radiology, 149 rue de Sèvres, 75015 Paris, France; e-mail: Volodia.dangouloff-ros@aphp.fr

Indicates article with supplemental on-line photo.

http://dx.doi.org/10.3174/ajnr.A6740

AJNR Am J Neuroradiol 41:1733-39 Sep 2020 www.ajnr.org 
follow-up duration (mean time interval generally $<4$ years ${ }^{11-14}$ ) or a relatively small number of patients and/or MR imaging examinations. Most studies suggested that FASI tend to decrease in size during adolescence. ${ }^{8,11,13-17}$ However, these children may also develop confounding MR images because they are at risk of developing low-grade gliomas within but also outside the optic pathways. Therefore, knowing the expected growth and vanishing pattern of FASI is essential to correctly assess abnormalities on MR imaging in these children.

Moreover, previous studies reported signal change within the hippocampi, which led to some discordance among authors because some have described diffuse and bilateral hyperintensities as a distinct lesion, ${ }^{16}$ while others have considered these hyperintensities to be FASI. ${ }^{2,18,19}$ Knowing the characteristics and temporal evolution of these signal changes in comparison with other FASI could help to conclude whether they should be considered the same pathologic entity.

In the current study, we retrospectively reviewed serial $M R$ imaging findings in a cohort of children with confirmed NF1 who had long-term MR imaging follow-up. We aimed to quantify the temporal evolution of FASI according to their regional distribution and sex, using a wide series of MR imaging examinations. In addition, we examined hippocampal intensity on T2WI and T2 FLAIR sequences.

\section{MATERIALS AND METHODS}

We followed the Strengthening the Reporting of Observational Studies in Epidemiology guidelines ${ }^{20}$ for this retrospective observational study. Local institutional review board authorization was granted (EDRACT 2014-A-00541-46), and the requirement for written informed consent was waived.

\section{Population and Procedures}

We performed a retrospective review of the NF1 data base of Necker Enfants Malades Hospital, Paris, France, from January 2007 to December 2018. Inclusion criteria were the following: children younger than 18 years of age at the time of their first MR imaging, with a diagnosis of NF1, using the National Institutes of Health Consensus criteria ${ }^{21}$ and having undergone $\geq 2$ brain MR imaging examinations at least 4 years apart. To avoid bias in FASI evaluation, we excluded children with a history of radiation therapy or surgery involving the ROI due to the potential for major posttherapeutic sequelae.

\section{MR Imaging Acquisition}

MR imaging equipment and techniques varied in the cases with the longest follow-up, and some of the patients underwent brain MR imaging at outside institutions before referral to our unit. All MR imaging examinations at our institution (67\% of the cohort) were performed using a Signa HDxt $1.5 \mathrm{~T}$ system (GE Healthcare) and a 12-channel head-neck-spine coil.

Our older brain MR imaging protocol included 2D sequences acquired in the axial plane: T2WI spin-echo sequence (TR/ $\mathrm{TE}=7522 / 117 \mathrm{~ms}$, section thickness $=4 \mathrm{~mm}$, intersection gap $=0.4 \mathrm{~mm}$, voxel size $=1.3 \mathrm{~mm}, 2$ excitations) and a T2-
FLAIR spin-echo sequence $(\mathrm{TR} / \mathrm{TE}=9000 / 2250 / 150 \mathrm{~ms}$, section thickness $=4 \mathrm{~mm}$, intersection gap $=0.4 \mathrm{~mm}$, voxel size $=1.2 \mathrm{~mm}, 2$ excitations). All measurements were preferentially made on the T2-FLAIR sequence unless it was of insufficient quality.

In recent years, $3 \mathrm{D}$ sequences became standard practice in our institution: 3D T2WI spin-echo sequences $(\mathrm{TR} / \mathrm{TE}=2500 / 102$ $\mathrm{ms}, \mathrm{FOV}=512 \times 512 \mathrm{~mm}^{2}$, section thickness $=1 \mathrm{~mm}$, intersection gap $=0.5 \mathrm{~mm}$, flip angle $=90^{\circ}$ ) or 3D-T2-FLAIR-weighted spin-echo sequences $(\mathrm{TR} / \mathrm{TE}=8400 / 2234 / 166 \mathrm{~ms}, \mathrm{FOV}=512 \times$ $512 \mathrm{~mm}^{2}$, section thickness $=1.2 \mathrm{~mm}$, intersection gap $=$ $0.6 \mathrm{~mm}$, flip angle $=90^{\circ}$ ).

\section{Image Analysis}

We used a medical image viewer. Two radiologists, a junior radiologist (S.C. with 4 years of experience) and an experienced neuroradiologist (R.L. with 7 years of experience), analyzed each case in consensus.

FASI were defined by visual inspection as areas of T2WI or T2-FLAIR hyperintensity greater than normal gray matter, without mass effect, contrast enhancement, or restriction on DWI.

Counting and surface area measurements were performed manually by 1 observer (S.C.) by drawing irregular ROIs in the axial or coronal planes, with the resulting surface area computed by the image viewer. If FASI were present on $>1$ contiguous section, an individual lesion was measured once on the section where it appeared the largest. FASI were categorized by size, according to their maximal diameter ( 5 groups defined as $<5 \mathrm{~mm}$; 5-10 mm; 10-15 mm; 15-20 mm; >20 mm) in each anatomic region (7 clusters in infra- and supratentorial regions, both left and right sides: capsular lenticular, caudate nucleus, thalamus, corpus callosum, cerebral white matter, brain stem, and cerebellum), reflecting the pattern of FASI distribution in previous reports.

Each surface area (in square millimeters) represented the addition of all FASI surface areas within a given anatomic region. Total lesion number and surface area per region for each MR imaging of all patients were recorded.

For the analysis of the hippocampi, a hyperintensity was defined as an area of intensity higher than that of the cortex, as assessed by the 2 readers in consensus. The presence of a hyperintensity, its unilateral/bilateral location, diffuse/focal features, and the parameters of the corresponding sequence $(2 \mathrm{D}, 3 \mathrm{D}, \mathrm{T} 2 \mathrm{WI}$, T2-FLAIR) have been listed above.

\section{Statistics}

All analyses were performed using SAS software, Version 9.4 of the SAS System for Windows (SAS Institute).

Descriptive analyses were performed by sex and overall for the total number, total surface area, and number by size for each of the 10 considered anatomic regions (overall, 2 zones [infraand supratentorial], 7 clusters). For each of the considered regions and each of the 5 size classes, the total number in the class was counted and descriptive qualitative analyses were performed by sex and for the overall cohort. 


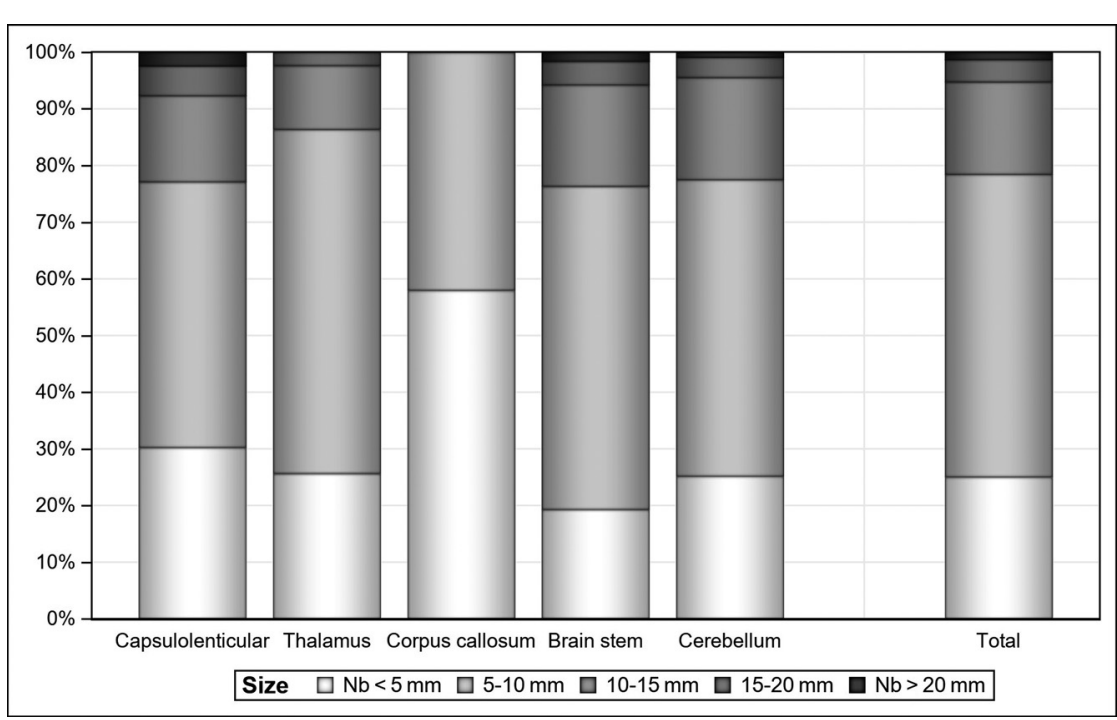

FIG 1. The distribution of FASI on all MR imaging examinations in size classes by clusters. Seventy-nine percent of FASI were $<10 \mathrm{~mm}$. FASI of $>20 \mathrm{~mm}$ appeared only for females $(n=7$ females, 2 of whom were outliers) and only in the capsular lenticular region, brain stem, and cerebellum clusters.

Due to the presence of outliers, some analyses were performed with and without them (3 patients whose total surface area was $>750 \mathrm{~mm}^{2}$ ). Some analyses were performed including a linear regression model between the number of lesions and surface area, using a cubic B-spline to fit a smooth curve through a scatterplot of age versus surface area, and some regressions, stepwise or not, between patient age and the total surface area.

A generalized linear mixed model ("proc mixed" in SAS) was used to take into account the repetition of MRIs in patients and the nonequally spaced time intervals between MR imaging examinations (repeated statement with spatial power) including effects for age, age ${ }^{2}$, age ${ }^{3}$, sex, and the 3 interactions, sex with age, age $^{2}$ age $^{3}$. Each analysis was performed interactively as a stepwise analysis: First, all effects were included in the model, and for each subsequent step, the effect with the higher $P$ value was excluded and so on until all $P$ values were $<.05$. The final model retained is the model with the effects for age, age $^{2}$, age ${ }^{3}$ (sex and all interactions are not significant); and from the final regression model with outliers, 2 ages of interest were calculated, the apex and the nadir of the surface area.

\section{RESULTS}

\section{Patients and MR Imaging}

Of the 53 patients who met the inclusion criteria, 14 were excluded because of surgical sequelae. No patient had a prior history of radiation therapy. Among the included patients, 23 had an optic pathway glioma (optic nerve, $n=18$; optic chiasm, $n=5$ ). No patient had a tumor outside the optic pathways or any mass effect or contrast enhancement outside the optic chiasm.

This study included 39 pediatric patients ( 22 females and 17 males, 56\% and 44\%) with an age range from 11 months to
13.7 years at the first available brain MR imaging examination (mean, 4.7 years; 95\% CI, 3.8-5.6 years) and 5.4-18.8 years at the last available MR imaging (mean, 12.1 years; 95\% CI, 11.0-13.3 years). They had undergone between 2 and $22 \mathrm{MR}$ imaging examinations (mean, 7.3; 95\% CI, 5.8-8.8) during a time interval from 4 to 14.4 years (mean, 7.4 years; 95\% CI, 6.6-8.2 years).

From these 39 patients, $285 \mathrm{MR}$ imaging examinations were available for review (female $=186, \quad 65.3 \%$; male $=99,34.7 \%)$. Of these, there were 181 2D-T2WI sequences (63.5\%), 190 2D-T2-FLAIR sequences (67\%), 77 3D-T2WI sequences (27\%), and 47 3D-T2 FLAIR sequences (16,5\%). Seventy-four percent (211/285) of MRIs contained both T2WI and T2 FLAIR sequences. All patients had at least 1 examination with contrast media injection, but details of gadolinium molecule and dose were not available due to the long retrospective follow-up.

\section{Number and Size of FASI Overall in the Study}

The frequency of patients with FASI was $100 \%$ in our cohort. Among them, 4 patients had no FASI seen on one of their MR imaging examinations ( 4 patients: 0.9 years, 1.5 years, 2.8 years, and 15.0 years of age; 4/285 [1.4\%] of studied MR images).

Overall, 3268 FASI were counted, $65.2 \%$ in the infratentorial region, of which $35.0 \%$ were in the cerebellum; $30.3 \%$, in the brain stem; $21.5 \%$, in the capsular lenticular region; $11.7 \%$, in the thalamus; $1.5 \%$, in the corpus callosum; and none in the caudate or white matter supratentorial clusters. Overall, 53.4\% were of $5-10 \mathrm{~mm} ; 25.1 \%,<5 \mathrm{~mm} ; 16.3 \%, 10-15 \mathrm{~mm} ; 3.9 \%$, 15-20 mm; and 1.3\%, > $20 \mathrm{~mm}$ (Fig 1).

\section{Number, Size, and Surface Area of FASI across Time}

A linear relationship between the number of FASI and the total surface area was observed with an $R^{2}$ of 0.83 . The scatterplots of number and surface area of FASI (Fig 2 and On-line Figure) show an appearance of FASI starting at 1 year, followed by an increase until the apex at about 7 years of age, followed by a decrease until the nadir at about 17-18 years of age (Fig 3). The mean age of the patients at the individual peak of FASI surface area was 7.2 years (Fig 4 ).

Among the 9 children younger than 2 years of age at first MR imaging, 3 did not have FASI (mean age, 1.7 years). These appeared in the time interval before the second MR imaging, performed at a mean age of 3.5 years.

The interpretation of the results for the group 16-19 years of age must be made with caution because only 7 MRIs for 5 patients were available (female $=1$, male $=4$ ). Contrary to the impression given by the curve, these patients showed a further decrease in or stability of their number of FASI/FASI surface areas, with the 

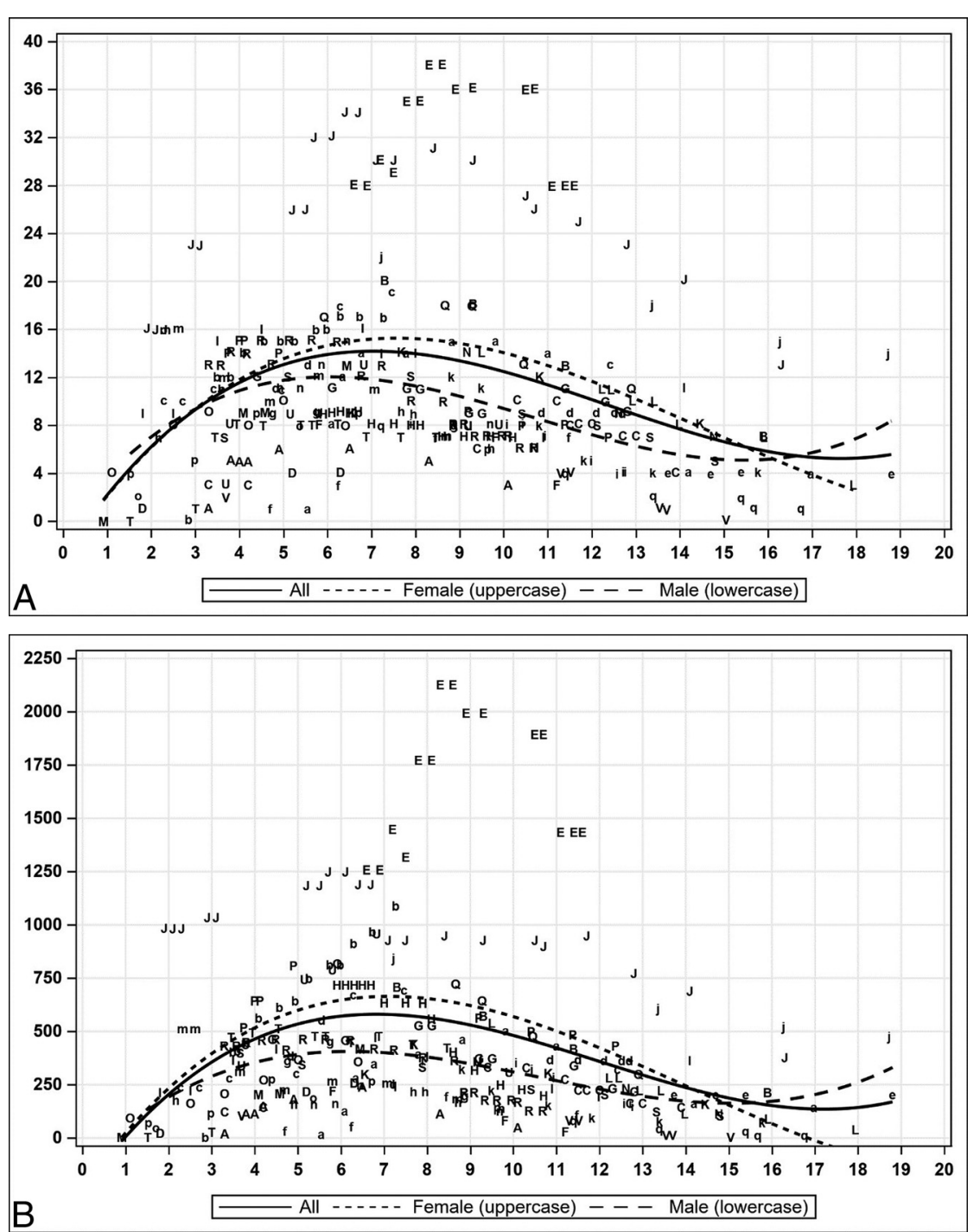

FIG 2. Temporal evolution of scatterplots of the total FASI number of all MRIs (A) and total FASI surface area of all MRIs (B). Uppercase letters are for females, and lowercase letters are for males. The regression curve is $3^{\circ}$. The $x$-axis represents age, the $y$-axis represents the number of FASI $(A)$, and the surface area is in square millimeters $(B)$. Female and male regression curves are descriptive.

apparent statistical increase being explained by the disappearance of patients with lower lesion load in the cohort.

The shapes of the regression curves were similar for the infratentorial region and flatter for the supratentorial region. From the complete regression model, no effect of sex was observed $(P=.76)$. The same analysis without outliers ( 3 patients with total FASI surface area of $>750 \mathrm{~mm}^{2}$ ) showed very similar results with an apex of 6.6 years and a nadir of 16.5 years. The same analysis performed on all MRIs for each anatomic region led to the same interpretation: The apex varied between 6.4 and 7.5 years, and the nadir, between 16.0 to 18.8 years.

\section{Hippocampal Analysis}

Of the 39 patients, 23 had a hippocampal hyperintensity $(\mathrm{HH})$ on at least $1 \mathrm{MR}$ imaging. The 23 patients included in this subgroup for analysis (59\%, 12 female and 11 male) accounted for 163 MRIs (time interval $=7.6$ years; $95 \% \mathrm{CI}, 3.7-14.4$ years; average $\mathrm{MR}$ imaging/patient $=$ 7; 95\% CI, 2-22; mean age $=8.7$ years; 95\% CI, 0.9-18.8 years). This subgroup included $55 \%$ of the total number of girls and $65 \%$ of the total number of boys without statistical difference $(P=.74)$.

The HHs were diffuse, bilateral, and symmetric (100\%) (Fig 5). The 23 patients with $\mathrm{HH}$ had a higher total surface area of FASI than the 16 patients without $\mathrm{HH}$ (mean, $394 \mathrm{~mm}^{2}$; 95\% CI, $0-1245 \mathrm{~mm}^{2}$ versus mean, $507 \mathrm{~mm}^{2}$; 95\% CI, $0-2123 \mathrm{~mm}^{2} ; P=$ $.03)$.

Eighteen of 23 patients had $\mathrm{HH}$ on their first MR imaging (mean age, 6.2 years; 95\% CI, 1.7-13.7 years), whereas 5 of 23 patients had normal hippocampi on their initial MR imaging. The $\mathrm{HH}$ appeared on follow-up MR imaging (mean age, 4.2 years; $95 \%$ CI, 2.6-6.7 years). No MR imaging performed before 3 years of age $(n=$ 9) showed $\mathrm{HH}$.

Eleven of 23 patients had at least 1 follow-up MR imaging with negative findings (mean age, 11.8 years; 95\% CI, 6.5-17 years), and this consistently corresponded to a transition from $2 \mathrm{D}$ to $3 \mathrm{D}$ sequences. $\mathrm{HH}$ did not disappear on follow-up MR imaging with $2 \mathrm{D}$ sequences, including after 11 years of age.

Of the 163 MRIs, 112 MRIs included a 2D-T2-FLAIR sequence, of which 84 (75\%) showed $\mathrm{HH}$ and 24 included a 3D-T2-FLAIR sequence, of which 2 (8\%) showed HH. One hundred MRIs comprised both 2D-T2WI and 2D-T2-FLAIR sequences, of which 75 (75\%) showed $\mathrm{HH}$ only on the 2D-T2-FLAIR sequences.

\section{DISCUSSION}

We report the neuroimaging findings in a large MR imaging study with long-term follow-up of children with NF1 and demonstrate the evolution pattern of FASI with time.

The frequency of patients with FASI was $100 \%$, which is greater than all previously published results in the literature, probably because of the inclusion criterion of a 4-year follow-up period and also because our institution is a neurosurgical tertiary referral center with a probable selection bias toward symptomatic patients.

The spatial distribution of FASI in our series is in agreement with that of previously published data, ${ }^{2,12,13,17,22-24}$ involving, in 
order of decreasing frequency, the cerebellum, brain stem, capsulolenticular region, and thalamus. Some reports did not specifically identify involvement of the thalamus but combined it with other anatomic regions. ${ }^{11,15}$ Because FASI were not frequently observed in the caudate nucleus, corpus callosum, or supratentorial white matter, the occurrence of $\mathrm{T} 2$ hyperintensities in these

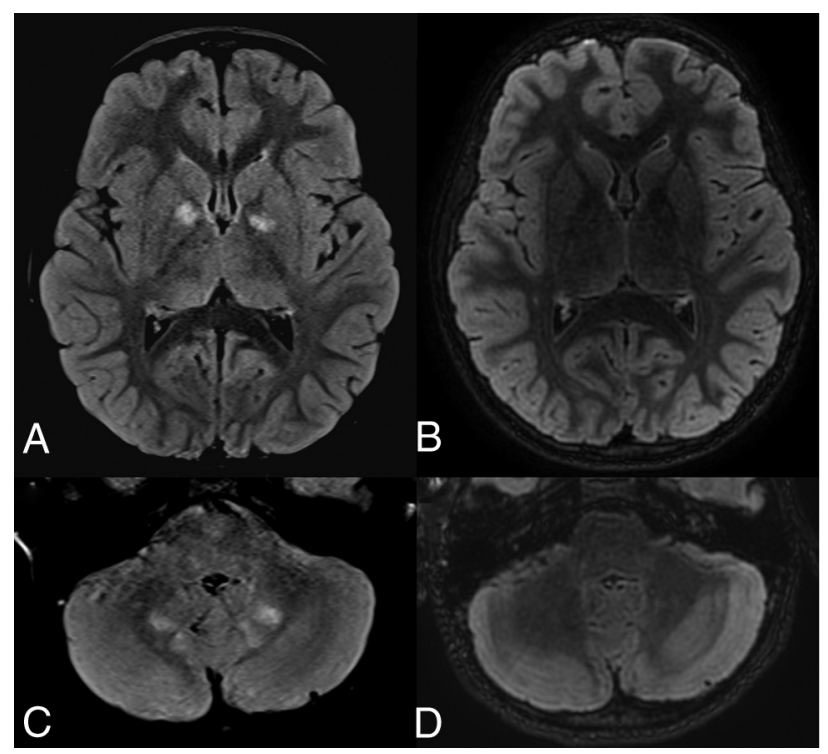

FIG 3. Temporal evolution of FASI on T2-FLAIR-weighted images. Axial T2-FLAIR sequences show capsular lenticular $(A)$ and infratentorial white matter (C) FASI in a 6-year-old patient, then follow-up MR imaging in a 10-year-old patient with almost complete disappearance of these FASI ( $B$ and $D)$.

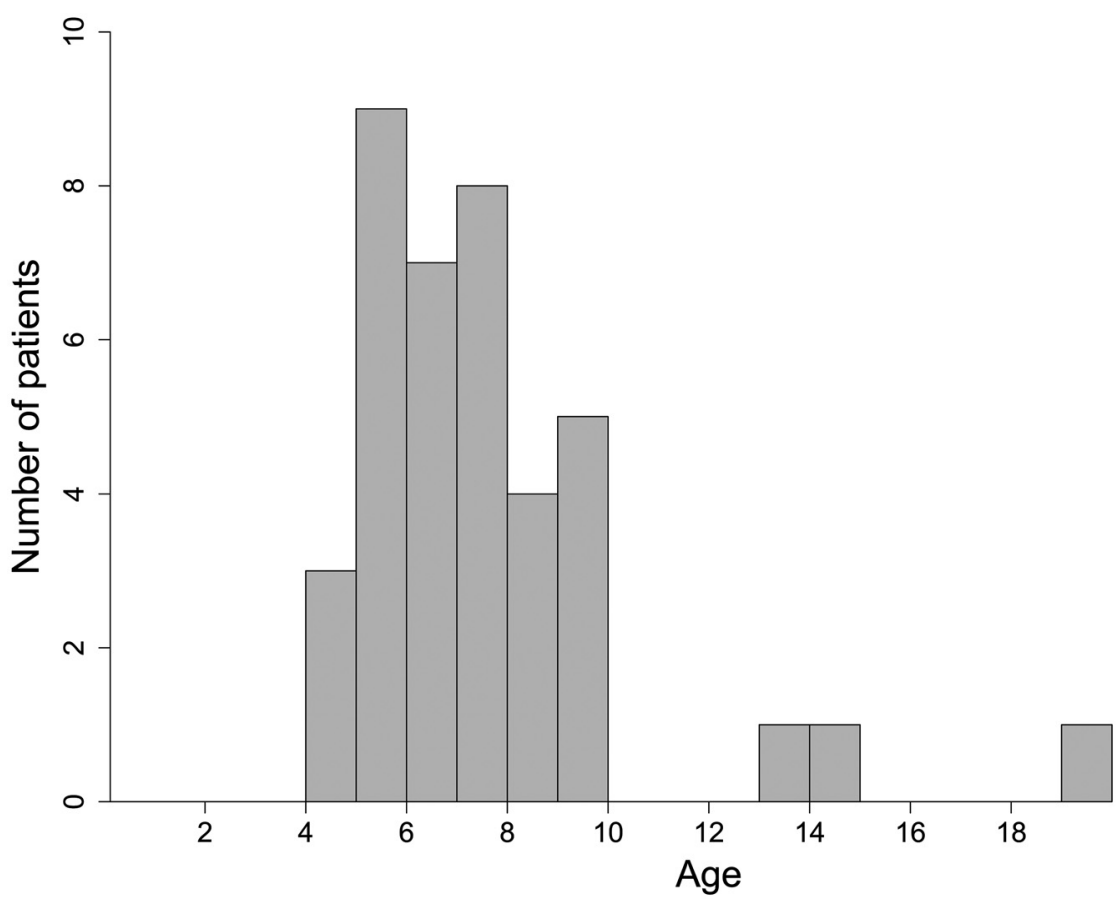

FIG 4. Age of the patients at the time of the peak of FASI surface. The mean age at the peak was $7.2 \pm 2.8$ years. The 3 patients with a peak age after 13 years had their first MR imaging after the age of 7 years $(7.5,13$, and 13 years). localizations could be a red flag for an atypical lesion, including a tumoral lesion, not a classic FASI.

In agreement with a previous report, ${ }^{14}$ FASI size was generally $<10 \mathrm{~mm}$. Other authors noted that FASI were $15 \mathrm{~mm}^{25}$ or that the maximum lesion diameter was $25 \mathrm{~mm} .{ }^{24}$ Therefore, in the setting of larger FASI, while they may correspond to a confluence of many FASI, an underlying tumoral process cannot be excluded and should be followed up.

No FASI displayed a mass effect or contrast enhancement, and none eventually developed into a glioma. This finding may be explained by our exclusion criteria, which included surgery involving the ROI at any time during the study period. Radiologists should exercise caution when the presence of a mass effect, with or without contrast enhancement, is detected in relation to a FASI because these features can correspond to low-grade or even high-grade gliomas outside the optic pathways in patients with NF1. ${ }^{26,27}$

Our results confirm the findings from previous studies that FASI are transient and dynamic abnormalities. First, in our cohort, FASI appeared from 1 year of age. Among the studies that included children younger than 4 years, ${ }^{2,15,24,25}$ none specified the age at the appearance of FASI.

Second, we found that an increase in size or de novo appearance of lesions is expected in young patients, with growth of characteristic FASI occurring until 7 years of age. The absence of such findings in other studies may reflect differences in methods because we did not have any predefined age group. In fact, most authors have studied patient groups consisting of age classifications with arbitrary thresholds, such as 10 years, ${ }^{13,22,24}$ or 4 and 12 years, ${ }^{17}$ or 15 years. ${ }^{1,11}$ Other authors ${ }^{18}$ analyzed brain MR imaging from children in age groups, particularly 4.0-6.9 and 7.0-9.9 years, and showed that most children accumulate FASI before 7 years of age, with a tendency toward FASI stabilization after that age (in the age range of 7.0-9.9 years). Furthermore, the larger size of our cohort and the longer follow-up of individual patients may better explain the age accuracy in our results.

Finally, after reaching the apex, FASI decrease until adulthood (nearly 17 years). Some authors concluded that FASI decrease from the beginning of adolescence $2,7,12,16$ or that they increase in number and size until 12 years of age. ${ }^{2}$ However, our results did not show such an association with puberty because we found that FASI tend to decrease earlier in childhood and are not correlated to sex. Some studies $^{12,16}$ included children older than 8 years of age, directly after the apex in our study, corresponding to the period when FASI have already started to decrease in number and size. Our data provide evidence of 


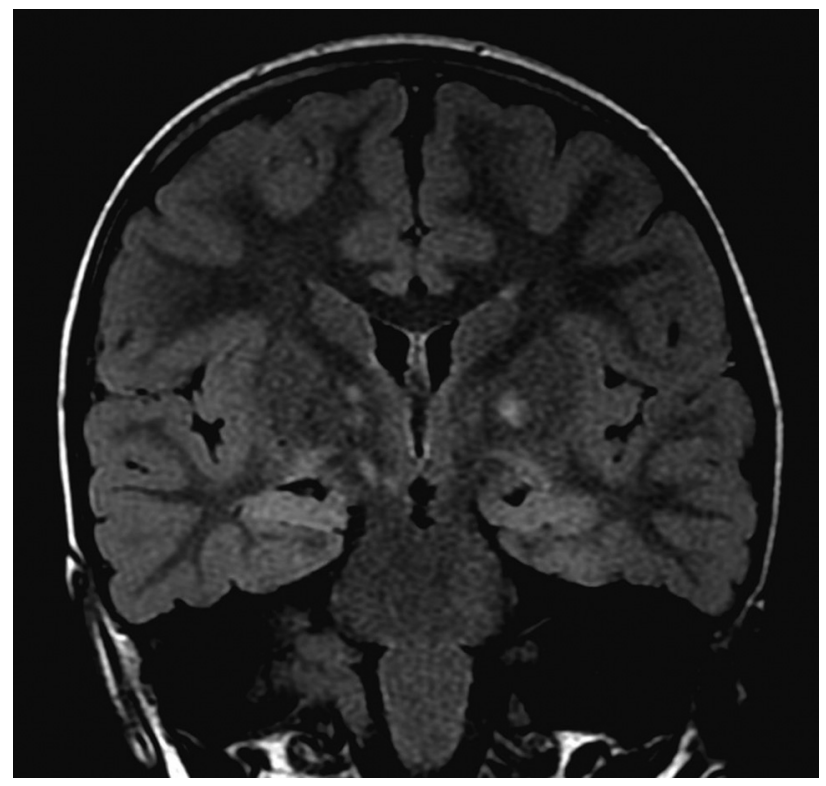

FIG 5. Hippocampal hyperintensity on T2 FLAIR image. Coronal T2 FLAIR sequence shows the diffuse homogeneous and symmetric hyperintensity of both hippocampi. FASI are also seen in the globus pallidus on both sides.

dissociation between the evolution of FASI and puberty. These findings indicate the necessity to evaluate the relationship between this evolution pattern and clinically determined pubertal status.

We did not find any difference between the evolution of anatomic regions and clusters, contrary to a previous report ${ }^{11}$ that concluded that FASI in the cerebellum decreased earlier than those in the brain stem and basal ganglia. Likewise, 2 distinct patterns have been reported, ${ }^{12}$ a linear decrease of FASI in the cerebellar hemisphere of adolescents and a nonlinear appearance/ regression in the basal ganglia region, with an increase again in late adolescence.

Given the high frequency and specificity of the presence of FASI in NF1, some authors ${ }^{2,23}$ recommended the inclusion of the presence of FASI as a diagnostic criterion for NF1 in children. It has been reported that the differential diagnosis for high-signal foci found in the cerebral white matter of $1.9 \%$ of healthy patients $^{28}$ versus those found in patients with NF1 should not be a problem because they have different characteristics and different anatomic predilections. ${ }^{14}$ Because we demonstrate the temporal evolution of FASI, using them as a diagnostic criterion could be of interest for patients between 4 and 12 years of age but would not be very useful for early childhood or adolescence.

\section{Hippocampal Analysis}

There are few data on hippocampal abnormalities in NF1. Hippocampal hyperintensities have been described as bilateral and diffuse, present in $80 \%$ of 1 cohort $^{16}$ and easier to see on T2 FLAIR. Our study revealed diffuse, bilateral, and symmetric hippocampal hyperintensities in nearly $60 \%$ of patients.

Because we found no correlation between the temporal evolution and the number of FASI and that the total surface area of FASI was lower in patients with $\mathrm{HH}$, our results would suggest that hippocampal hyperintensity is a separate entity from FASI, contrary to other studies ${ }^{2,18,19}$ that considered the hippocampi as an anatomic region affected by FASI.

These signal abnormalities seem to depend on the technique used because the hyperintensity appears more frequently on the 2D-T2-FLAIR sequences compared with the 3D-T2-FLAIR and 2D-T2WI sequences. Moreover, the disappearance of these hyperintensities seems to be related to the change of technique and not to the age of the patients. 3D-T2-FLAIR seemed to be inferior to 2D-T2-FLAIR in terms of lesion contrast and conspicuity in the hippocampus, such as in patients with hippocampal sclerosis. ${ }^{29}$ This feature could be explained by the higher signal of the normal hippocampus on 3D-T2-FLAIR; radiologists should be aware of this finding. FASI should not have this pitfall because 3D-T2-FLAIR has been shown to be equal or superior to 2D-T2FLAIR for other gray or white matter lesions. ${ }^{29}$ The subjectivity of these diffuse and symmetric signal changes and the dependence on the technique used may limit the clinical impact of this feature in patients with NF1. A potential link with clinical findings and the underlying pathophysiology remains to be demonstrated.

\section{Limitations}

Our study has some limitations, including selection bias. Our institution is a tertiary referral center that receives a high proportion of symptomatic or atypical patients. Furthermore, a minimum follow-up of 4 years increases the frequency of MR imaging findings. Patients with a history of surgery were excluded; therefore, our evolution criteria do not apply to them.

All of our data pertain to brain MR imaging at $1.5 \mathrm{~T}$, which limits the applicability of our results to MR imaging at 3T. It seems unlikely that this would have modified the longitudinal variations of FASI, but the analysis of hippocampal hyperintensity may be different at $3 \mathrm{~T}$.

Most patients underwent a combination of both 2D and 3D MR imaging sequences during the course of their follow-up, which could affect the variability of surface area measurements. However, we were unable to analyze this effect because the patients did not have $2 \mathrm{D}$ and $3 \mathrm{D}$ sequences at the same time. The surface area measurements of FASI were similar between T2WI and T2 FLAIR sequences. Because of the low number of 3D sequences, we were unable to use volumetric measurements for follow-up (instead of surface area), which could have been a more accurate way to measure FASI. 2D section thickness was high $(4 \mathrm{~mm})$, possibly hampering the accuracy of measurements of FASI using these sequences. Because our inclusion criteria mandated a long-term follow-up period and due to the larger size of our cohort, the inclusion of multiple different MR imaging techniques was unavoidable. As imaging becomes ever more sensitive, it will be increasingly important to define a standardized imaging protocol for brain MR imaging in the setting of NF1.

\section{CONCLUSIONS}

Our study, based on a large sample of MR imaging examinations with long-term follow-up of patients with NF1, provides a more complete view of FASI evolution.

Having demonstrated a predictable pattern of longitudinal variation of FASI in children with NF1, we suggest that FASI are 
not related to puberty or adolescence because they decrease at an earlier age, from 7 years, and there is no significant difference according to sex. However, we did not assess pubertal status at the time of each MR imaging examination, precluding drawing any strong conclusions about this potential correlation. These results should be subject to future prospective studies with larger sample sizes and knowledge of pubertal status, which could lead to an improved understanding of FASI pathophysiology.

We also found that hippocampal hyperintensities frequently seen in patients with NF1 are not correlated with FASI evolution, supporting the hypothesis of a different process, which still needs to be elucidated.

We are convinced that a better understanding of FASI could have positive practical implications. A "watch and wait" approach could be implemented until the age of apex, provided no atypical imaging sign is present (mass effect, contrast enhancement). Knowledge of the predicted evolution allows more accurate prognostic advice to be conveyed to the referring physician and family during follow-up.

Disclosures: François Doz-UNRELATED: Board Membership: Bayer, Bristol Myers Squibb, Celgene, Loxo, Roche, Servier, Tesaro*; Consultancy: Servier*; Grants/ Grants Pending: Synth Innove*; Payment for Development of Educational Presentations: Bristol Myers Squibb*; Travel/Accommodations/Meeting Expenses Unrelated to Activities Listed: Bayer, Bristol Myers Squibb, Roche. *Money paid to the institution.

\section{REFERENCES}

1. Nicita F, Di Biasi C, Sollaku S, et al. Evaluation of the basal ganglia in neurofibromatosis type 1. Childs Nerv Syst ChNS Syst 2014;30:319-25 CrossRef Medline

2. Lopes Ferraz Filho JR, Munis MP, Soares Souza A, et al. Unidentified bright objects on brain MRI in children as a diagnostic criterion for neurofibromatosis type 1. Pediatr Radiol 2008;38:305-10 CrossRef Medline

3. North KN, Riccardi V, Samango-Sprouse C, et al. Cognitive function and academic performance in neurofibromatosis 1: consensus statement from the NF1 Cognitive Disorders Task Force. Neurology 1997;48:1121-27 CrossRef Medline

4. DiPaolo DP, Zimmerman RA, Rorke LB, et al. Neurofibromatosis type 1: pathologic substrate of high-signal-intensity foci in the brain. Radiology 1995;195:721-24 CrossRef Medline

5. Billiet T, Mädler B, D'Arco F, et al. Characterizing the microstructural basis of "unidentified bright objects" in neurofibromatosis type 1: a combined in vivo multicomponent $\mathrm{T} 2$ relaxation and multi-shell diffusion MRI analysis. Neuroimage Clin 2014;4:649-58 CrossRef Medline

6. Ferner RE, Chaudhuri R, Bingham J, et al. MRI in neurofibromatosis 1: the nature and evolution of increased intensity $\mathrm{T} 2$ weighted lesions and their relationship to intellectual impairment. J Neurol Neurosurg Psychiatry 1993;56:492-95 CrossRef Medline

7. Hyman SL, Gill DS, Shores EA, et al. T2 hyperintensities in children with neurofibromatosis type 1 and their relationship to cognitive functioning. J Neurol Neurosurg Psychiatry 2007;78:1088-91 CrossRef Medline

8. Payne JM, Pickering T, Porter M, et al. Longitudinal assessment of cognition and T2-hyperintensities in NF1: an 18-year study. Am J Med Genet A 2014;164:661-65 CrossRef

9. Feldmann R, Schuierer G, Wessel A, et al. Development of MRI T2 hyperintensities and cognitive functioning in patients with neurofibromatosis type 1. Acta Paediatr Oslo Paediatr 2010;99:1657-60 CrossRef Medline
10. Ottenhoff MJ, Rietman AB, Mous SE, ENCORE-NF1 Team, et al. Examination of the genetic factors underlying the cognitive variability associated with neurofibromatosis type 1. Genet Med 2020; 22:889-97 CrossRef Medline

11. Itoh T, Magnaldi S, White RM, et al. Neurofibromatosis type 1: the evolution of deep gray and white matter MR abnormalities. AJNR Am J Neuroradiol 1994;15:1513-19 Medline

12. Kraut MA, Gerring JP, Cooper KL, et al. Longitudinal evolution of unidentified bright objects in children with neurofibromatosis-1. Am J Med Genet A 2004;129A:113-19 CrossRef Medline

13. Menor F, Marti-Bonmati L, Arana E, et al. Neurofibromatosis type 1 in children: MR imaging and follow-up studies of central nervous system findings. Eur J Radiol 1998;26:121-31 CrossRef Medline

14. Raininko R, Thelin L, Eeg-Olofsson O. Non-neoplastic brain abnormalities on MRI in children and adolescents with neurofibromatosis type 1. Neuropediatrics 2001;32:225-30 CrossRef Medline

15. DiMario FJ, Ramsby G. Magnetic resonance imaging lesion analysis in neurofibromatosis type 1. Arch Neurol 1998;55:500-05 CrossRef Medline

16. Gill DS, Hyman SL, Steinberg A, et al. Age-related findings on MRI in neurofibromatosis type 1. Pediatr Radiol 2006;36:1048-56 CrossRef Medline

17. Khan A, Beri S, Baheerathan A, et al. Globus pallidus high-signal lesions: a predominant MRI finding in children with neurofibromatosis type 1. Ann Indian Acad Neurol 2013;16:53-56 CrossRef Medline

18. Griffiths PD, Blaser S, Mukonoweshuro W, et al. Neurofibromatosis bright objects in children with neurofibromatosis type 1: a proliferative potential? Pediatrics 1999;104:e49 CrossRef Medline

19. Yamanouchi $H$, Kato $T$, Matsuda $H$, et al. MRI in neurofibromatosis type I: using fluid-attenuated inversion recovery pulse sequences. Pediatr Neurol 1995;12:286-90 CrossRef Medline

20. von Elm E, Altman DG, Egger M, et al. The Strengthening the Reporting of Observational Studies in Epidemiology (STROBE) statement: guidelines for reporting observational studies. Lancet 2007;370:1453-57 CrossRef

21. Neurofibromatosis: Conference Statement-National Institutes of Health Consensus Development Conference. Arch Neurol 1988;45:575-78 Medline

22. Ferraz-Filho JR, José da Rocha A, Muniz MP, et al. Unidentified bright objects in neurofibromatosis type 1 : conventional MRI in the follow-up and correlation of microstructural lesions on diffusion tensor images. Eur J Paediatr Neurol 2012;16:42-47 CrossRef Medline

23. DeBella K, Poskitt K, Szudek J, et al. Use of "unidentified bright objects" on MRI for diagnosis of neurofibromatosis 1 in children. Neurology 2000;54:1646-51 CrossRef Medline

24. Sevick RJ, Barkovich AJ, Edwards MS, et al. Evolution of white matter lesions in neurofibromatosis type 1: MR findings. AJR Am J Roentgenol 1992;159:171-75 CrossRef Medline

25. Aoki S, Barkovich AJ, Nishimura K, et al. Neurofibromatosis types 1 and 2: cranial MR findings. Radiology 1989;172:527-34 CrossRef Medline

26. Campian J, Gutmann DH. CNS tumors in neurofibromatosis. J Clin Oncol 2017;35:2378-85 CrossRef Medline

27. Spyris CD, Castellino RC, Schniederjan MJ, et al. High-grade gliomas in children with neurofibromatosis type 1 : literature review and illustrative cases. AJNR Am J Neuroradiol 2019;40:366-69 CrossRef Medline

28. Dangouloff-Ros V, Roux C-J, Boulouis G, et al. Incidental brain MRI findings in children: a systematic review and meta-analysis. AJNR Am J Neuroradiol 2019;40:1818-23 CrossRef Medline

29. Kakeda S, Korogi Y, Hiai Y, et al. Pitfalls of 3D FLAIR brain imaging: a prospective comparison with 2D FLAIR. Acad Radiol 2012; 19:1225-32 CrossRef Medline 